Proceedings of the ASME 2021 40th International

Conference on Ocean, Offshore and Arctic Engineering

OMAE2021

June 21-30, 2021, Virtual, Online

\title{
OMAE2021-61525
}

\section{ADVANCED INTELLIGENT CONTROL STRATEGY IN DYNAMIC POSITIONING (DP) SYSTEM APPLIED TO A SEMI-SUBMERSIBLE DRILLING PLATFORM IN THE NORTH SEA}

\author{
Mohamad Alremeihi \\ Newcastle University \\ Newcastle, UK
}

\author{
Rosemary Norman \\ Newcastle University \\ Newcastle, UK
}

\author{
Kayvan Pazouki \\ Newcastle University \\ Newcastle, UK
}

\author{
Arun Dev \\ Newcastle University \\ Singapore, Singapore
}

\author{
Musa Bashir \\ Liverpool John Moores University \\ Liverpool, UK
}

\begin{abstract}
Dynamic Positioning (DP) systems play a crucial role in oil and gas drilling and production floaters used globally for deepwater operations. Drilling operations need to maintain automatic positioning of the platform in the horizontal-plane within the safe zone. Operating DP systems typically require highly responsive control systems when encountering prevailing weather conditions. However, DP incident analysis demonstrates that control and thruster failures have been the leading causes of accidents for the past two decades, according to the International Marine Contractors Association (IMCA). In this paper, a Predictive Neural Network (PNN) strategy is proposed for thruster allocation on a platform; it has been developed by predicting the platform response and training the network to transform the required force commands from a nonlinear Proportional Integral Derivative (PID) motion controller for each thruster. The strategy is developed for increasing safety and zone keeping of DP-assisted-drilling operations in harsh weather. This is done by allowing the platform to recover the position more rapidly whilst decreasing the risk of losing the platform position and heading, which can lead to catastrophic damage. The operational performance of the DP system on a drilling platform subjected to the North Sea real environmental conditions of wind, currents and waves, is simulated with the model incorporating the PNN control algorithm, which deals with dynamic uncertainties, into the unstable conventional PID control system for a current drilling semi-submersible model. The simulation results demonstrate the improvement in DP accuracy and robustness for the semi-
\end{abstract}

submersible drilling platform positioning and performance using the PNN strategy.

Keywords: Dynamic Positioning (DP), Semi-submersible drilling platform, Time Domain Simulation, Three Degree of Freedom (DOF) motion, DP advanced control system, Predictive Neural Network (PNN), Thruster Allocation control.

\section{INTRODUCTION}

In recent years, there has been growing interest in developing dynamic positioning (DP) systems due to increasing oil and gas demands and the need to extract these from deep waters and in extreme weather. Over the last four decades, the offshore oil and gas industry achieved growth with low risk by use of new technologies. A DP system can be defined as a computer-controlled system to calculate the necessary thrust force that automatically maintains the drilling platform position and heading at a fixed location by using the thruster system to counteract the environmental forces of wind, sea current and waves [1].

The first DP systems were implemented for horizontal mode operation in the 1960s and early 1970s, using single-input singleoutput PID control and Kalman filter theory-based methods. Nonlinear DP controller designs such as fuzzy logic, backstepping, and sliding mode control were later suggested [2]. Due to the nonlinearity of the DP control problem [3], modern techniques have used advanced control with intelligent behaviour and computational approaches such as adaptive nonlinear PID control, adaptive fuzzy logic theory and artificial neural networks (ANN). 
As DP technology became well established, studies focused on intelligent methods and the modification of performance for drilling operations. For the over-actuated optimization problem, the thruster forces needed by the DP system are distributed by a thruster allocation algorithm which should be precise, efficient and robust. Therefore, the thruster allocation algorithm should handle important issues such as power efficiency, wear and tear minimization, input saturation and rate limit, and thruster fault tolerance [4]. The main thrust allocation strategies are deterministic, pseudo-inverse matrix, nonlinear constraints optimization, genetic algorithms and sequential quadratic programming (SQP).

The DP incidents analysis conducted by the IMCA shows that DP control and thruster system failures represent a high percentage (around 30-40\%) of the leading causes of loss of position, which necessitates attention and further research [5]. The necessity of enhancing offshore operation safety by keeping the platform in the safe operating zone has been proven over time by the occurrence of major accidents. The consequences of DP drilling accidents are incredibly high, with significant damage to the facilities and environment and the possibility of crew fatalities.

This paper aims to build a reliable DP control system by integrating the PNN thruster allocation into an existing basic control system to perform maximum dynamic positioning, enhance the accuracy of the control and minimize the risks of losing the position. In this analysis, the conventional nonlinear PID motion control and the Moore-Penrose pseudo-inverse thruster allocation methods were used as the first stage of the research to compare with the PNN strategy.

In drilling operations, the DP system needs a quick response to apply precise amounts of force in specific directions to maintain the position and heading in harsh weather, and keep calculating all environmental force and direction data. The main objective is to increase the precision and reliability of DP drilling operations by achieving optimum thruster allocation force and direction while maintaining safe drilling operations. The addition of artificial intelligence methods to the thruster allocation control can make the system faster and more accurate than the DP operator response during drilling operations.

The paper is structured as follows: Section 2 presents a semi-submersible platform model, defining the mathematical model used. Section 3 explains the PNN allocation control design and demonstrates the numerical simulation. Section 4 presents the simulation results and a discussion of the outcomes. Finally, Section 5 concludes the study.

\section{SEMI-SUBMERSIBLE PLATFORM MATHEMATICAL MODELLING}

A method to enhance the accuracy and reliability of DP control by taking into account semi-submersible platform motion, environmental loads and thruster allocation by using PID motion and PNN thruster allocation controls is investigated in this paper. The vectorial notation of motion [6-7], is the most common method of modelling the motion of a vessel and has become the standard for marine control systems.

\subsection{Semi-submersible Drilling Platform Model}

With the data shown in Table 1 and using the DNV Sesam (GeniE) program, a hull model of an existing semi-submersible drilling platform has been designed, as shown in Figure 1. Using DNV Sesam (HydroD) and WAMIT, industry-standard software tools [8-9], hydrodynamic data for the platform model was created following DNV standards, to generate data required to simulate the platform behaviour.

TABLE 1: SEMI-SUBMERSIBLE PLATFORM DATA.

\begin{tabular}{cccc}
\hline LOA (m) & Width (m) & Draft (m) & Platform Mass (kg) \\
\hline 117 & 80 & 21 & $52,476,000$ \\
\hline
\end{tabular}

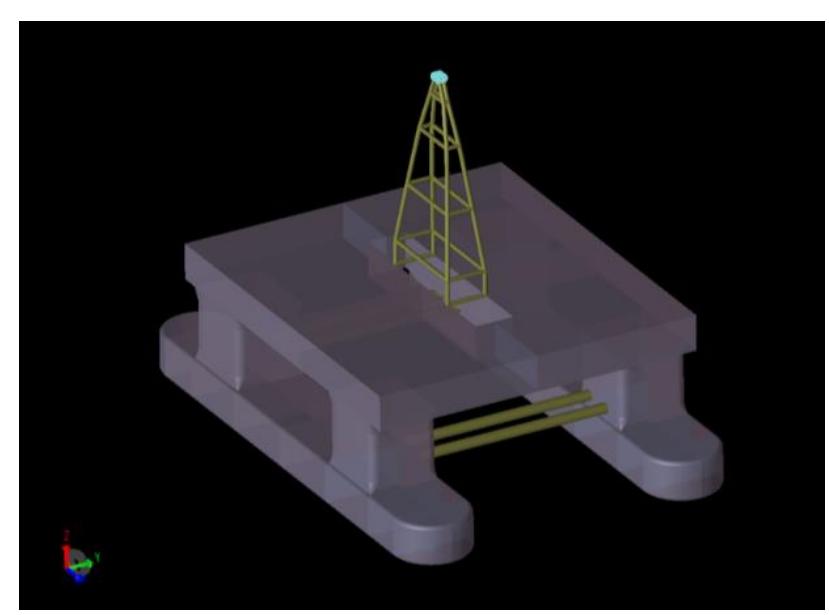

FIGURE 1: SEMI-SUBMERSIBLE PLATFORM HULL.

\subsection{Mathematical Modelling}

A mathematical model has been developed that explains the dynamics of the low frequency (LF) and wave frequency (WF) models of a drilling platform. The LF motions induce secondorder wave loads (drift forces including viscous effects and slowly varying forces), sea current loads, wind loads and forces of the thrusters. The WF causes the first-order wave loads. In this paper, it is found that it is suitable to consider the horizontalplane dynamics [10] assumed in modelling the positioning of marine control systems. Linear damping and wave drift loads usually form a large part of the over-all forces in the LF, which is again taken into account in this model. To explain the relationship between the control activity, the motion-induced and sea-keeping models to describe the motion due to the wave loads, the model is designed to compensate for WF and LF motions with a manoeuvring model. In general, wave loads of the first-order are more critical than wave loads of the secondorder; both can be calculated using quadratic transfer functions [11].

The study of dynamic equations of motion of the marine system can be divided into two aspects: the kinematic equations of motion relating to the geometric aspects, and the kinetic equations of motion corresponding to the motion analysis caused by the forces [7]. A specific mathematical model and parameters are needed for the drilling operational mode, as the primary physical properties will depend on how the platform is operated. 
The motions of the drilling platform in the horizontal-plane are in the directions of surge, sway and yaw; these are taken into consideration in the DP control. By using the generalized position $\eta$, defined with respect to an Earth-fixed reference frame (NED), and platform kinematics illustrated in Figure 2, the velocity $v$ is given with respect to a body-fixed frame, and $R(\psi)$ is the transformation matrix in a vectorial representation [7]. These six differential equations are lumped together into a three degree of freedom (DOF) reference frame $\eta=\left[\begin{array}{lll}x & y & \psi\end{array}\right]^{T}, v=$ $[u v r]^{T}$ as:

$$
\boldsymbol{R}(\boldsymbol{\psi})=\left[\begin{array}{ccc}
\dot{\operatorname{\eta }}= & R(\psi) v \\
\sin \psi & -\sin \psi & 0 \\
0 & 0 & 1
\end{array}\right]
$$

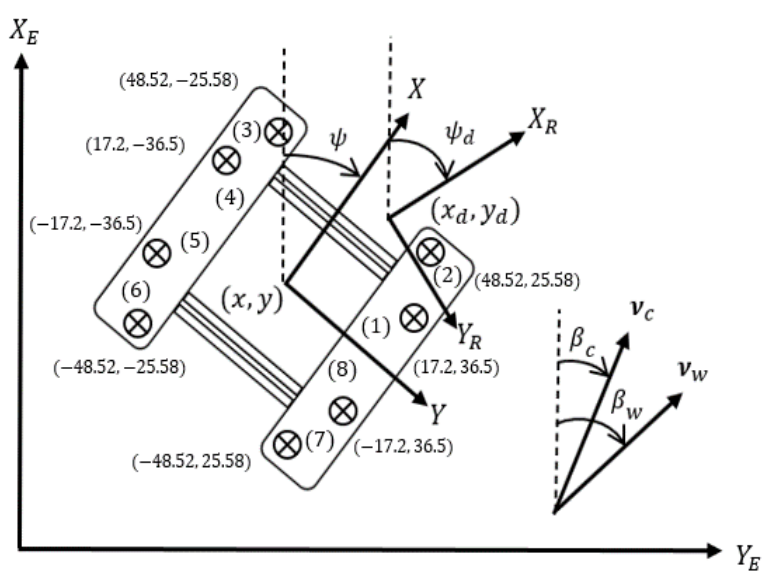

FIGURE 2: SEMI-SUBMERSIBLE PLATFORM KINEMATICS AND THRUSTERS' LOCATIONS (M).

In general, three differential equations, one for each degree of freedom, describe the rigid-body and hydrodynamic equations of motion. For the nonlinear unified sea-keeping and manoeuvring model, these three differential equations are lumped together into a vectorial equation of motion [7]:

$$
\begin{aligned}
& M \dot{v}+C_{R B}(v) v+C_{A}\left(v_{r}\right) v_{r}+D\left(v_{r}\right) v_{r}+G(\eta)= \\
& \tau_{\text {wind }}+\tau_{\text {wave } 1}+\tau_{\text {wave } 2}+\tau_{t h r}
\end{aligned}
$$

Where $M$ is the platform inertia matrix including the added mass, and $C_{R B}(v)$ is the skew-symmetric Coriolis and centripetal matrix. The effects of sea current on the platform are divided into two parts: the potential part is formulated as $C_{A}\left(v_{r}\right)$ which includes the Munk moments, and the viscous part [7]. The damping vector $D\left(v_{r}\right)$ is divided into linear and nonlinear components $D\left(v_{r}\right)=D_{L} v+D_{N L}\left(v_{r}, \gamma_{r}\right) . G(\eta)$ defines the restoring vector, $\tau_{\text {wind }}$ is the wind load vector, $\tau_{\text {wave } 1}$ is the first-order wave loads, $\tau_{\text {wave } 2}$ defines the second-order wave loads and $\tau_{t h r}$ represents the thruster forces. The platform inertia mass matrix $M$ includes added mass and the linear damping $D_{L} v$ and nonlinear damping $D_{N L}\left(v_{r}, \gamma_{r}\right)$ are defined as:

$$
\boldsymbol{M}=\left[\begin{array}{ccc}
m-X_{\dot{u}} & 0 & 0 \\
0 & m-Y_{\dot{v}} & -Y_{\dot{r}} \\
0 & -N_{\dot{u}} & I_{Z}-N_{\dot{r}}
\end{array}\right]
$$

Where $\mathrm{m}$ is the platform mass, $I_{Z}$ is the moment of inertia about the z-axis, and $X_{\dot{u}}, Y_{\dot{v}}, Y_{\dot{r}}, N_{\dot{u}}$ and $N_{\dot{r}}$ are the zerofrequency added mass in the surge, sway and yaw directions; hence, $M$ is symmetrical and positive definite.

$$
\boldsymbol{D}_{\boldsymbol{L}} \boldsymbol{v}=\left[\begin{array}{ccc}
-X_{u} & 0 & 0 \\
0 & -Y_{v} & -Y_{r} \\
0 & -N_{v} & -N_{r}
\end{array}\right]
$$

$D_{L} v$ defines the strictly positive damping caused by linear wave drift and laminar skin friction damping, where $X_{u}, Y_{v}, Y_{r}$, $N_{v}$ and $N_{r}$ are the hydrodynamic potential damping can be calculated by the DNV Sesam (HydroD) tool.

$$
\boldsymbol{D}_{\boldsymbol{N} \boldsymbol{L}}\left(\boldsymbol{v}_{\boldsymbol{r}}, \gamma_{\boldsymbol{r}}\right)=0.5 \rho_{w}\left[\begin{array}{l}
L_{P P} D C_{c x}\left(\gamma_{r}\right) \\
L_{P P} D C_{c y}\left(\gamma_{r}\right) \\
L_{P P} D C_{c \psi}\left(\gamma_{r}\right)
\end{array}\right]
$$

The nonlinear damping $D_{N L}\left(v_{r}, \gamma_{r}\right)$ needs $C_{c x}, C_{c y}$ and $C_{c \psi}$ which are the non-dimensional drag coefficients in the horizontal plane, and can be found by model tests or using the DNV Sesam (SIMO) software for the platform with some defined location of the origin. In Equation (6) $\rho_{w}$ is the water density, $L_{P P}$ is the platform length between perpendicular, and $D$ is the platform drilling draft. The motions are usually nonlinear, but it is possible to measure linear approximations of particular points. Furthermore, the sea current loads typically included in the LF model define the relative velocity vector $v_{r}$ and drag angle $\gamma_{r}$ according to:

$$
\begin{gathered}
\boldsymbol{v}_{\boldsymbol{r}}=\left[u_{r} v_{r} r\right]^{T}=\left[u-u_{c} v-v_{c} r\right]^{T} \\
\boldsymbol{\gamma}_{r}=\operatorname{atan} 2\left(u_{r}, v_{r}\right)
\end{gathered}
$$

The horizontal sea current formulation in surge and sway are defined as $u_{c}$ and $v_{c}$ noting that in yaw $r$ is small almost equal to zero, where $\nu_{c}$ and $\beta_{c}$ are the sea current speed and direction, respectively as follows:

$$
\begin{gathered}
\boldsymbol{u}_{\boldsymbol{c}}=v_{c} \cos \left(\beta_{\boldsymbol{c}}-\psi\right) \\
\boldsymbol{v}_{\boldsymbol{c}}=v_{c} \sin \left(\beta_{\boldsymbol{c}}-\psi\right) \\
\boldsymbol{v}_{\boldsymbol{c}}=\sqrt{u_{r}{ }^{2}+v_{r}{ }^{2}}
\end{gathered}
$$

The formulation of the wind velocities $u_{w}$ and $v_{w}$ are defined according to the following, the total wind speed $v_{w}$, and relative wind angle $\gamma_{w}$ maybe simplified to:

$$
\begin{gathered}
\boldsymbol{u}_{\boldsymbol{w}}=v_{w} \cos \left(\beta_{w}-\psi\right) \\
\boldsymbol{v}_{\boldsymbol{w}}=v_{w} \sin \left(\beta_{w}-\psi\right) \\
\boldsymbol{v}_{\boldsymbol{w}}=\sqrt{u_{w}{ }^{2}+v_{w}{ }^{2}}
\end{gathered}
$$




$$
\gamma_{w}=\beta_{w}+\psi
$$

The wind loads $\tau_{\text {wind }}$ in the surge, sway and yaw directions are defined as follows:

$$
\boldsymbol{\tau}_{\text {wind }}=0.5 \rho_{a} v_{w}{ }^{2}\left[\begin{array}{c}
A_{w x} D C_{w x}\left(\gamma_{w}\right) \\
A_{w y} D C_{w y}\left(\gamma_{w}\right) \\
A_{w y} D C_{w \psi}\left(\gamma_{w}\right) L_{o a}
\end{array}\right]
$$

Where $\rho_{a}$ is the air density, $L_{o a}$ is the platform length overall, and $A_{w x}, A_{w y}$ are the lateral and longitudinal areas of the platform freeboard projected on the xz-plane and yz-plane. $C_{w x}\left(\gamma_{w}\right), C_{w y}\left(\gamma_{w}\right)$ and $C_{w \psi}\left(\gamma_{w}\right)$ are the non-dimensional wind coefficients in the horizontal plane which can be calculated by DNV Sesam (SIMO) software. From assuming the linear force superposition $\tau_{\text {wave } 1}$ and $\tau_{\text {wave } 2}$ are obtained by using the Quadratic transfer function to get the Response Amplitude Operator (RAO) forces [7] for the panel model using the WAMIT software tool, as shown in Figure 3.

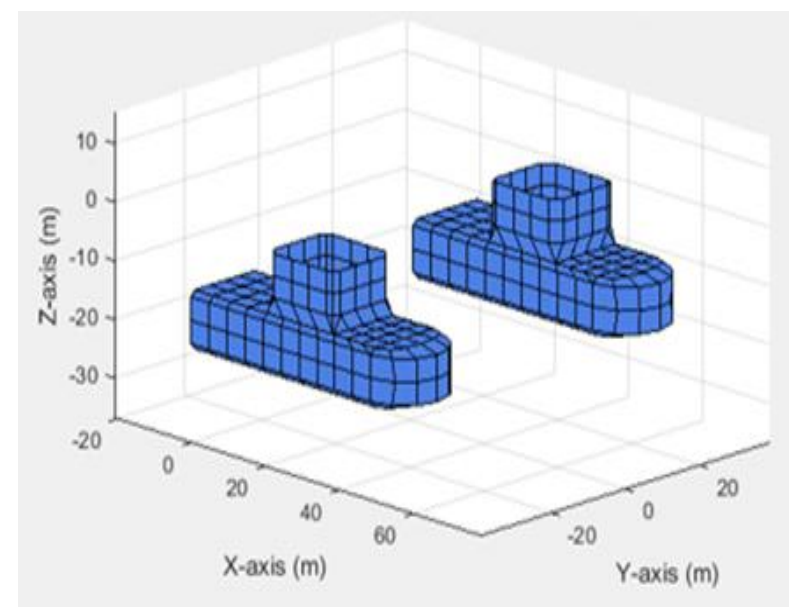

FIGURE 3: SEMI-SUBMERSIBLE PLATFORM PANEL MODEL.

$$
\begin{gathered}
\boldsymbol{\tau}_{\boldsymbol{t h r}}=\left[\begin{array}{c}
X_{t h r} \\
Y_{\boldsymbol{t h r}} \\
N_{t h r}
\end{array}\right] \\
=T_{3 \times 8}(\alpha) f_{1 \times 8}=T_{3 \times 8}(\alpha) K_{8 \times 8} u_{1 \times 8}
\end{gathered}
$$

$\tau_{t h r}$ defines the generalized forces generated by the thruster system, and the thrust configuration $T_{3 \times 8}(\alpha)$ with the azimuth angles $\alpha$, the control forces $f=K u$ with the magnitudes of the force produced by each thruster vector $u$, and $K$ is a diagonal force coefficient matrix. $T_{3 x 8}(\alpha)$ can be expressed by the following,

$$
\boldsymbol{T}_{\boldsymbol{i}}\left(\boldsymbol{\alpha}_{\boldsymbol{i}}\right)=\left[\begin{array}{c}
\cos \alpha_{i} \\
\sin \alpha_{i} \\
l_{x i} \cos \alpha_{i}+l_{y i} \sin \alpha_{i}
\end{array}\right]
$$

Where the angle $\alpha_{i}$ is the angle of the $i$-th actuator $i=$ $1 \ldots 8$, determining the force direction produced in the platform body-fixed coordinate system, and $l_{x i}, l_{y i}$ are the locations of the thrusters in the platform, as shown in Figure 2. By using the extended thrust vector, $\boldsymbol{\tau}_{\boldsymbol{c}}$ is defined according to [7]:

$$
\begin{array}{r}
\boldsymbol{\tau}_{c}=T_{e} f_{e}=T_{e} K u_{e}=\left[\begin{array}{cc}
1 & 0 \\
0 & 1 \\
-l_{y i} & l_{x i}
\end{array}\right] K u_{e} \\
u_{e}=\left[\begin{array}{ll}
u_{x i} & u_{y i} \mid
\end{array}\right]^{T}
\end{array}
$$

Where $u_{e}$ is the extended control input vector, and $T_{e}$ is the extended thrust configuration, which is constant while $T_{i}\left(\alpha_{i}\right)$ depends on $\alpha_{i} . u_{e}$ can be solved directly from Equation (19) using a least-squares optimization method, then the azimuth control can be derived from $u_{e}$ by mapping the pairs $\left(u_{x i}, u_{y i}\right)$ [12]:

$$
\begin{gathered}
\boldsymbol{u}_{i}=\sqrt{u_{x i}+u_{y i}} \\
\boldsymbol{\alpha}_{i}=\tan ^{-1}\left(\frac{u_{x i}}{u_{x i}}\right)
\end{gathered}
$$

With control inputs that are subject to both amplitude and rate saturation, the azimuth angles must be determined. The solution of this problem is defined as the generalized inverse through the use of Lagrange multipliers.

$$
\boldsymbol{T}_{\boldsymbol{e}}^{*}=W^{-1} T_{e}^{T}\left(T_{e} W^{-1} T_{e}^{T}\right)^{-1}
$$

In the case where $W=I$, this gives an equal weighting to each control force where $W$ is a positive definite matrix. Thus, Equation (23) reduces to the Moore-Penrose pseudo-inverse. Since $f_{e}=T_{e}^{*} \tau_{c}$, the control input can be computed as:

$$
\boldsymbol{u}_{e}=K^{-1} T_{e}^{*} \tau_{c}
$$

In the simulation, the semi-submersible platform was commanded to maintain the horizontal-plane position and heading. The nonlinear PID controller is modelled, using the reference system inputs for the platform to calculate the error signal $\eta_{e}$. The nonlinear PID motion control receives the error signals and provides the platform with the forces needed. The actual values for position and speed are derived from the dynamic equations of the platform.

$$
\boldsymbol{\tau}_{\boldsymbol{P I D}}=-K_{p} \eta_{e}-R(\psi) K_{d} v-K_{i} \int_{0}^{t} \eta_{e}(\tau) d \tau
$$

Where $K_{p}, K_{i}$ and $K_{d}$ are the non-negative controller gains for the proportional, the time integral and the time derivative of the error signal, respectively. The PID control has been designed and modelled using the marine systems simulator (MSS) toolbox for Simulink [13]. The controller's gains used for drilling operation were obtained through tuning using the Simulink Optimization toolbox as presented in Table 2. 
TABLE 2: NONLINEAR PID CONTROL GAINS

\begin{tabular}{cccc}
\hline & $\boldsymbol{X}$ & $\boldsymbol{Y}$ & $\boldsymbol{\psi}$ \\
\hline $\mathbf{K i}$ & $8.81 \mathrm{E}+07$ & $1.40 \mathrm{E}+10$ & $1.39 \mathrm{E}+07$ \\
$\mathbf{K p}$ & $4.50 \mathrm{E}+06$ & $7.02 \mathrm{E}+08$ & $7.00 \mathrm{E}+05$ \\
$\mathbf{K d}$ & $4.50 \mathrm{E}+04$ & $7.02 \mathrm{E}+06$ & $7.00 \mathrm{E}+03$ \\
\hline
\end{tabular}

\section{PREDICTIVE NEURAL NETWORK (PNN) MODEL}

Predictive neural network (PNN) control is an advanced method of control. A nonlinear DP control with a neural network (NN) model has been used to predict future platform performance using the optimization algorithm of NewtonRaphson to minimize the cost function. For a gradient-based iterative solution in real-time, the computationally efficient derivation of the PNN would minimize the cost function [14]. In three DOFs, the PNN provides the conversion between the necessary forces as input and the thruster commands as output. To optimize the platform output over a given time horizon, the PNN determines the thruster allocation control input. Figure 4 and Equation (26) demonstrate the NN procedure in which the number of inputs is multiplied by the weights, applied to the bias terms, and then passed through an activation function $\mathrm{F}(\mathrm{u})$.

$$
\boldsymbol{F}(\boldsymbol{u})=\sum_{j=1}^{N}\left(\omega_{j} y_{j}+\theta\right)
$$

where $\mathrm{N}$ is defined as the number of inputs, $\omega_{j}$ the weights of the input $\mathrm{j}, y_{j}$ is the input, and $\theta$ is the bias.

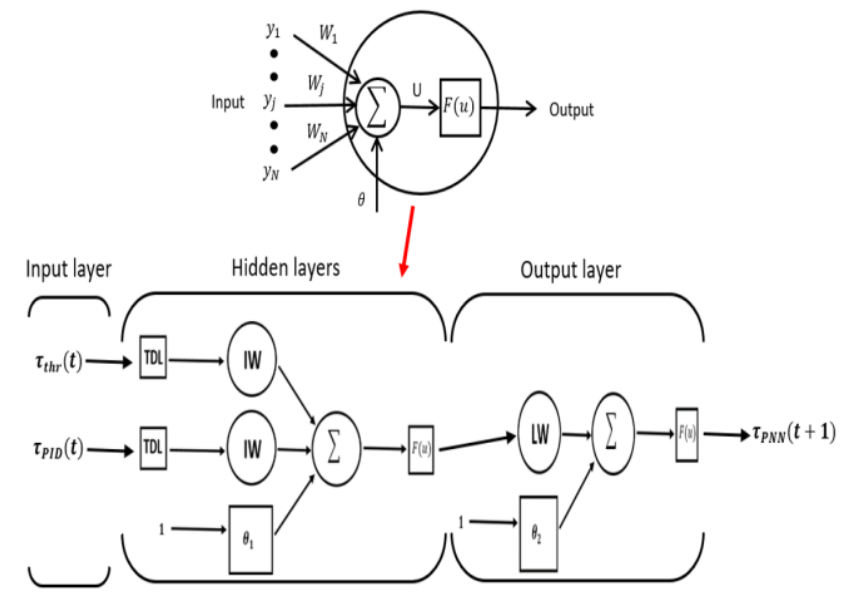

FIGURE 4: PNN MODEL STRUCTURE.

There is one hidden layer of tan-sigmoid transfer function (tan-sigmoid neurons) for the PNN multiple-layer feedforward networks with time-delayed line (TDL), including input weight (IW) and input bias $\theta_{1}$. This is followed by a linear transfer function output layer (linear function) used for the fitting problem, including layer weight $(\mathrm{LW})$ and output bias $\theta_{2}$. Multiple neuron layers with nonlinear transfer functions allow the network to learn nonlinear relationships between the PID control input and the thruster force output. This approach resulted in a PNN structure consisting of $3 \tau_{P I D}$ input neurons, 20 hidden layers and $8 u_{P N N}$ output neurons. The predictions were used by a numerical cost function optimization program to determine the control signal that would minimize the following output criterion over a specific horizon:

$$
\begin{gathered}
\boldsymbol{u}_{P N N}=\sum_{j=N_{1}}^{N_{2}}\left(\tau_{P I D}(t+j)-\tau_{P N N}(t+j)\right)^{2}+ \\
\rho \sum_{j=1}^{N_{u}}\left(u^{\prime}(t+j-1)-u^{\prime}(t+j-2)\right)^{2}
\end{gathered}
$$

where $N_{1}, N_{2}$, and $N_{u}$ describe the horizons over which the signal error and the control inputs are estimated. The term $u^{\prime}$ defines the tentative control signal that minimize the cost function of $u_{P N N} \cdot \tau_{P I D}$ describes the required response, and $\tau_{P N N}$ defines the neural network model response. The $\rho$ value reflects the effect on the output index of the sum of the squares of the control augmentations. To describe the forward dynamics of the platform thruster allocation, the first stage of PNN is to train the NN model. The prediction error between the thruster system outputs and the NN model outputs is used for training the signal. In order to determine the values of the tentative control signal, the PNN includes the NN model and optimization block, which reduces the performance criterion and then integrates the optimal control signal into the thruster allocation. This was simulated by using the predictive control toolbox for the neural network in the Simulink program. The overall simulation also includes three environment modules in which wind, sea current and waves are produced, as shown in Figure 5.

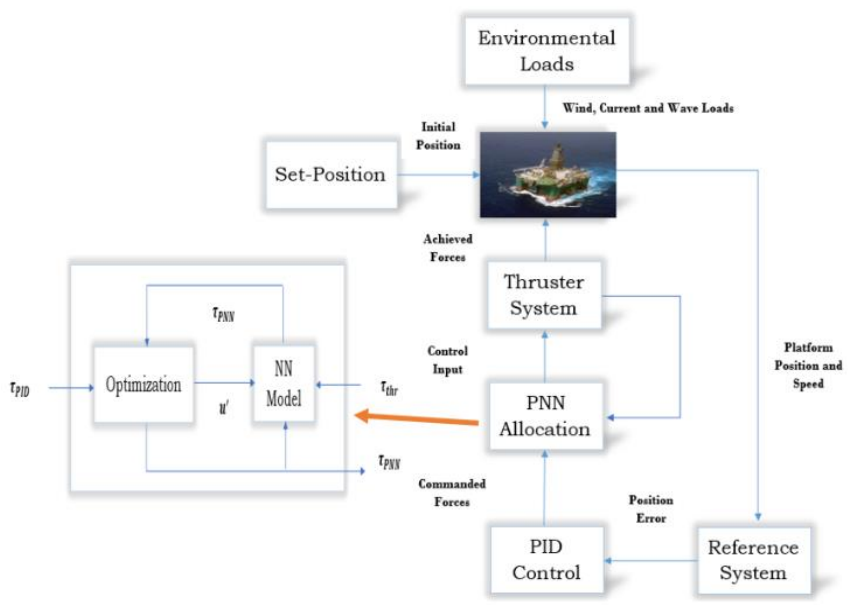

FIGURE 5: DP SIMULATION WITH PID MOTION AND PNN ALLOCATION CONTROLS.

The advanced DP motion control system is designed to regulate the LF and WF motions of a drilling platform based on five interconnected systems:

1) Set-position: set-position to the model of the platform so that the reference system calculates the position, course, and distance travelled that are required in turn to control the platform,

2) Reference System: the reference system to determine the position and heading errors to maintain the set-position,

3) PID Control: the PID motion control system to determine the required forces and moments of motion control produced by the platform to achieve a specific control objective, 
4) PNN Allocation: the PNN allocation control system that determines the thruster action needed to maintain the platform in a safe zone by optimizing the PID motion control signal by training the NN model to predict optimum performance using Bayesian regularization, and

5) Thruster System: in order to calculate the optimized control forces for each thruster, the thruster system generates the forces necessary to maintain the position and thruster allocation.

The simulation presented in this paper will analyse the possible operational impacts of the use of advanced control systems through three essential steps: first, the hull modelling of an existing semi-submersible drilling platform, using DNV Sesam (GeniE and HydroD), SIMO and WAMIT software, to measure hydrodynamic and environmental coefficient data. The second step in the modelling is to construct a time-domain simulation with nonlinear PID control, as in primary DP systems, following the method of Fossen (unified model) in which three DOFs are modelled in terms of manoeuvring and sea-keeping motion theories; this has been completed using Matlab/Simulink software. Third, by introducing the PNN control allocation, the DP control has been improved, and this has been developed using Simulink neural network predictive toolbox. The model simulates the environmental loads of wind, sea current, firstorder and second-order waves that impact the platform. The data is obtained from an integrated hydrodynamic and hydrostatic matrixes platform model (inertia mass, Coriolis centripetal, damping and restoring forces). The nonlinear PID motion control gets the difference in position and heading and sends the motion control input to the allocation of the thruster to configure the thruster force needed using the pseudo-inverse (Moore-Penrose). To provide the platform's required force, the PNN allocation control determines the best action to be sent to the thruster system. The system is tested by simulating DP drilling operations in 50 years probability extreme real weather conditions for the North Sea oil and gas deep-water fields on the Norwegian Continental Shelf (NCS), with environmental parameters [15] as shown in Table 3.

TABLE 3: ENVIRONMENTAL CONTROL LIMITATION.

\begin{tabular}{cccc}
\hline Wind $(\mathbf{m} / \mathbf{s})$ & Current $(\mathbf{m} / \mathbf{s})$ & Wave (m) & Depth (m) \\
\hline 32 & 1.1 & 10 & $300-700$ \\
\hline
\end{tabular}

\section{RESULTS AND DISCUSSION}

The system was simulated in a specific environmental, extreme sea condition for the semi-submersible model to investigate whether the platform could maintain its position in the drilling zone and the performance of the controls in 50-year probability North Sea condition. In the simulation, the semisubmersible platform was required to maintain position and heading at $x, y=0 \mathrm{~m}$ and $\psi=10 \mathrm{deg}$. The semi-submersible platform was modelled full scale in the DP simulation, but the diagrams show it at $10 \%$ of the actual size to better view the position and heading plots. Environmental angles of attack from the head, quarter and beam were tested using PID and PNN controls. To determine the safety of the DP drilling operation position and heading, safety zones were applied [16]. Drilling safety zones are defined as the Red region viewed as unacceptable, leading to an incident (28 $\mathrm{m}$ diameter), Yellow region viewed as caution and may lead to an incident $(22 \mathrm{~m}$ diameter) and the Green region viewed as satisfactory and safe for drilling operation (16 $\mathrm{m}$ diameter). The simulation results for the head, quarter and beam sea conditions for the position and heading of the semi-submersible platform using PID motion and PNN allocation controls are shown in Figures 6-8. The corresponding thruster force and angle efficiency are illustrated in Figure 9. Also, Tables 4-6 show the critical control performance parameters for the position and heading results. A comparison of the average and the standard deviation from the statistical analysis of the thruster force and angle is shown in Table 7.

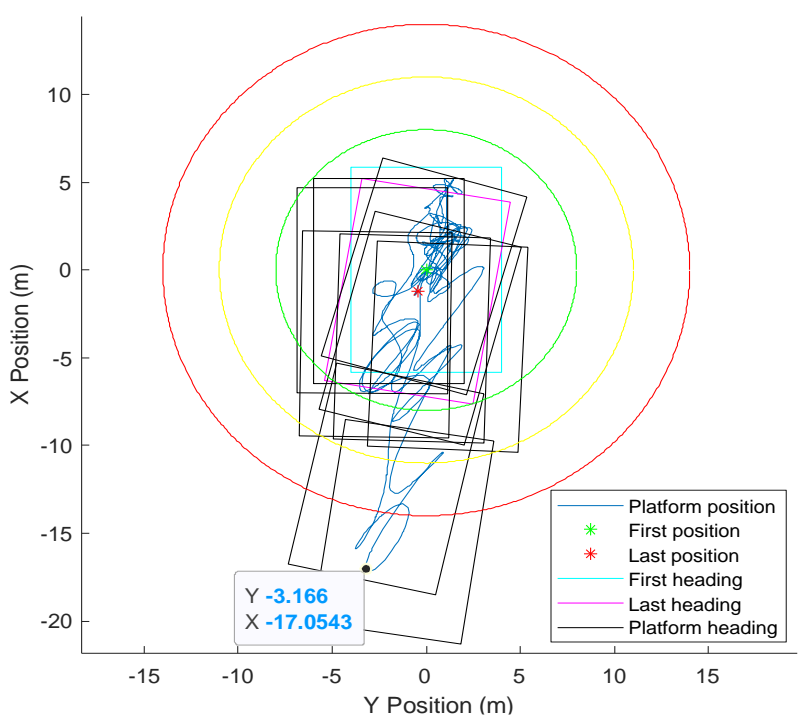

(a)

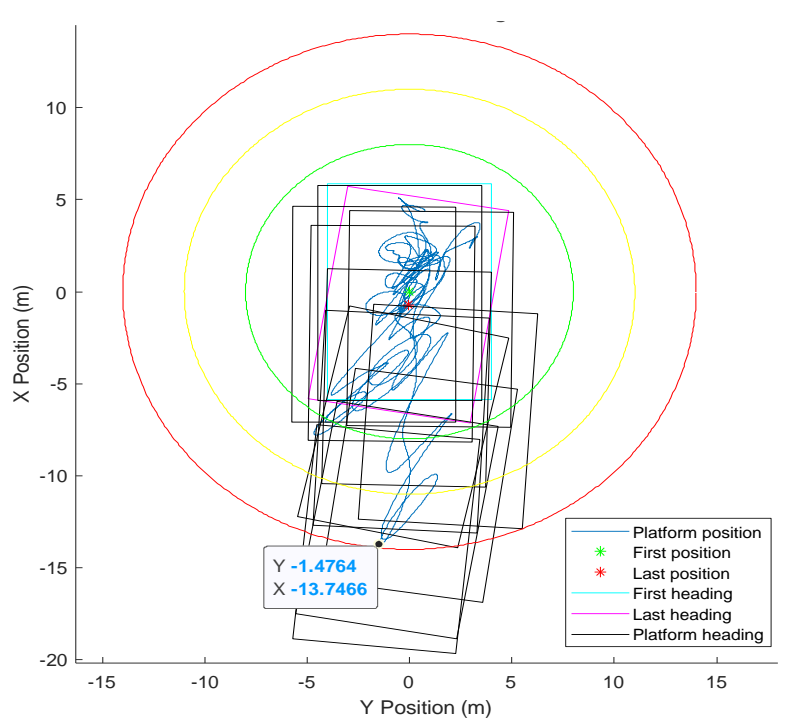

(b)

FIGURE 6: PLATFORM POSITION AND HEADING PLOT WITH PID AND PNN CONTROLS (HEAD SEA). 
TABLE 4: POSITION AND HEADING CONTROL PERFORMANCE DIFFERENCES (HEAD SEA).

\begin{tabular}{cccc}
$\begin{array}{c}\text { Position and } \\
\text { heading }\end{array}$ & $\mathbf{X}$ & $\mathbf{Y}$ & $\boldsymbol{\psi}$ \\
\hline \multicolumn{4}{c}{ PID } \\
\hline Peak time & $63.4 \mathrm{sec}$ & $75.7 \mathrm{sec}$ & $157.4 \mathrm{sec}$ \\
\hline Overshoot & $-17.1 \mathrm{~m}$ & $-3.7 \mathrm{~m}$ & $10 \mathrm{deg}$ \\
\hline Settling time & $277.5 \mathrm{sec}$ & $176.3 \mathrm{sec}$ & $402.1 \mathrm{sec}$ \\
\hline \multicolumn{4}{c}{ PID and PNN } \\
\hline Peak time & $76.2 \mathrm{sec}$ & $369 \mathrm{sec}$ & $157.3 \mathrm{sec}$ \\
\hline Overshoot & $-13.8 \mathrm{~m}$ & $-4.7 \mathrm{~m}$ & $9.9 \mathrm{deg}$ \\
\hline Settling time & $276.4 \mathrm{sec}$ & $134 \mathrm{sec}$ & $387.6 \mathrm{sec}$ \\
\hline
\end{tabular}

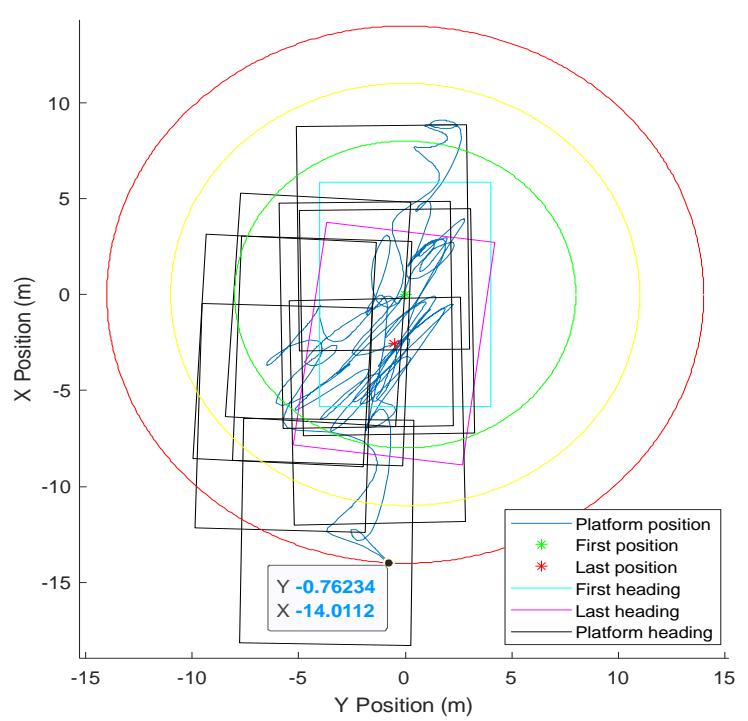

(a)

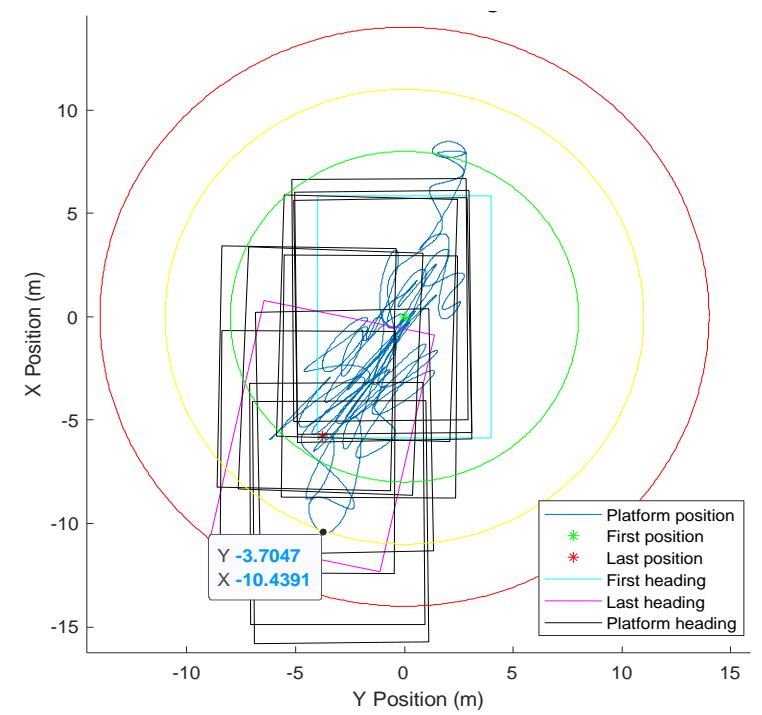

(b)

FIGURE 7: PLATFORM POSITION AND HEADING PLOT WITH PID AND PNN CONTROLS (QUARTER SEA).
TABLE 5: POSITION AND HEADING CONTROL PERFORMANCE DIFFERENCES (QUARTER SEA).

\begin{tabular}{cccc}
\hline $\begin{array}{c}\text { Position and } \\
\text { heading }\end{array}$ & $\mathbf{X}$ & $\mathbf{Y}$ & $\boldsymbol{\psi}$ \\
\hline \multicolumn{4}{c}{$\mathbf{P I D}$} \\
\hline Peak time & $55.1 \mathrm{sec}$ & $91.2 \mathrm{sec}$ & $276.4 \mathrm{sec}$ \\
\hline Overshoot & $-14 \mathrm{~m}$ & $-6.5 \mathrm{~m}$ & $21.1 \mathrm{deg}$ \\
\hline Settling time & $161.5 \mathrm{sec}$ & $174.1 \mathrm{sec}$ & $520 \mathrm{sec}$ \\
\hline \multicolumn{4}{c}{ PID and PNN } \\
\hline Peak time & $43.4 \mathrm{sec}$ & $241.4 \mathrm{sec}$ & $252.5 \mathrm{sec}$ \\
\hline Overshoot & $-10.4 \mathrm{~m}$ & $-6.2 \mathrm{~m}$ & $19.9 \mathrm{deg}$ \\
\hline Settling time & $159.6 \mathrm{sec}$ & $173 \mathrm{sec}$ & $456.6 \mathrm{sec}$ \\
\hline
\end{tabular}

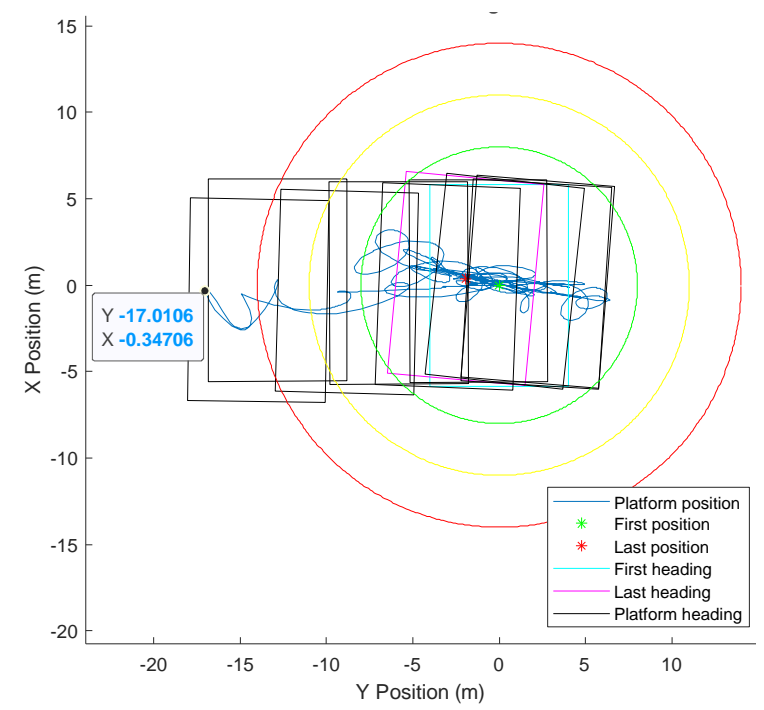

(a)

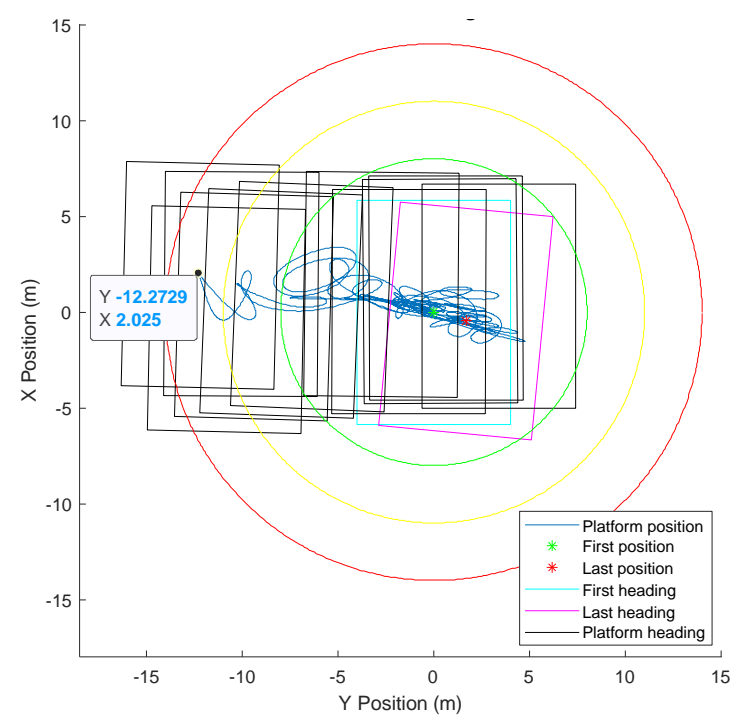

(b)

FIGURE 8: PLATFORM POSITION AND HEADING PLOT WITH PID AND PNN CONTROLS (BEAM SEA). 
TABLE 6: POSITION AND HEADING CONTROL PERFORMANCE DIFFERENCES (BEAM SEA).

\begin{tabular}{cccc}
\hline $\begin{array}{c}\text { Position and } \\
\text { heading }\end{array}$ & $\mathbf{X}$ & $\mathbf{Y}$ & $\boldsymbol{\psi}$ \\
\hline \multicolumn{4}{c}{ PID } \\
\hline Peak time & $341.5 \mathrm{sec}$ & $62.3 \mathrm{sec}$ & $332.6 \mathrm{sec}$ \\
\hline Overshoot & $3.2 \mathrm{~m}$ & $-17 \mathrm{~m}$ & $19.9 \mathrm{deg}$ \\
\hline Settling time & $119.6 \mathrm{sec}$ & $173 \mathrm{sec}$ & $780 \mathrm{sec}$ \\
\hline \multicolumn{5}{c}{ PID and PNN } \\
\hline Peak time & $355.4 \mathrm{sec}$ & $60.6 \mathrm{sec}$ & $347.6 \mathrm{sec}$ \\
\hline Overshoot & $3.4 \mathrm{~m}$ & $-12.3 \mathrm{~m}$ & $18.2 \mathrm{deg}$ \\
\hline Settling time & $139.6 \mathrm{sec}$ & $166.3 \mathrm{sec}$ & $720 \mathrm{sec}$ \\
\hline
\end{tabular}

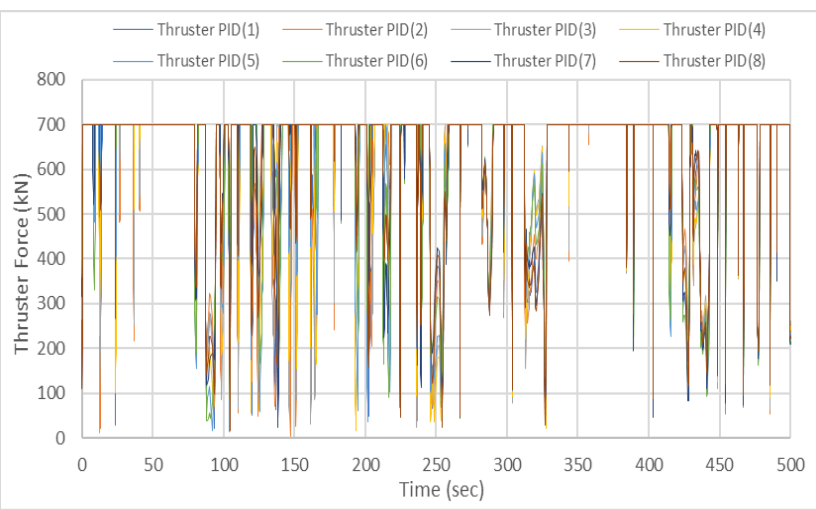

(a)

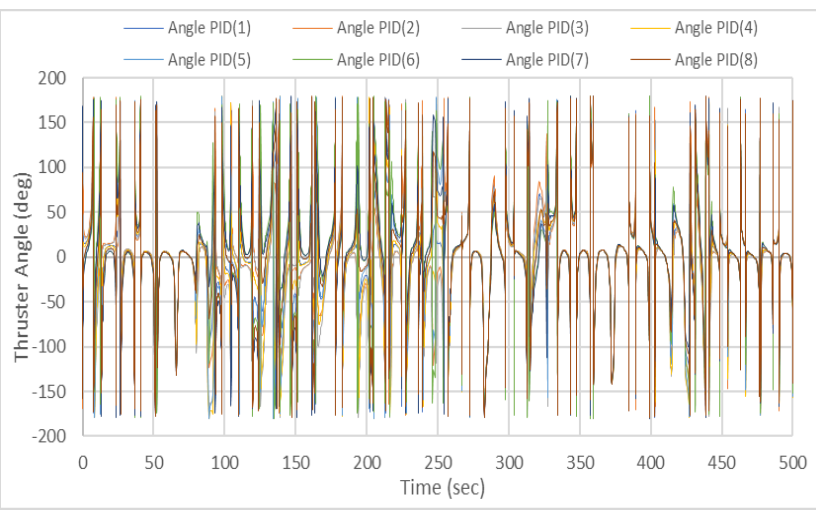

(b)

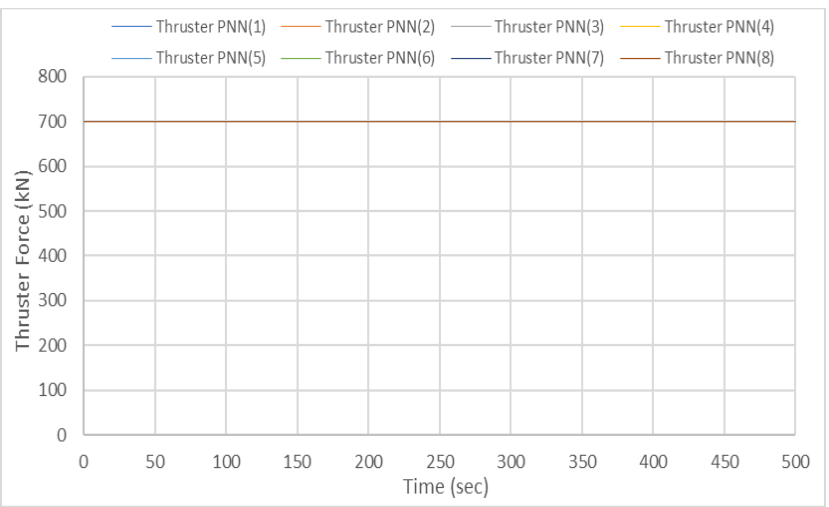

(c)

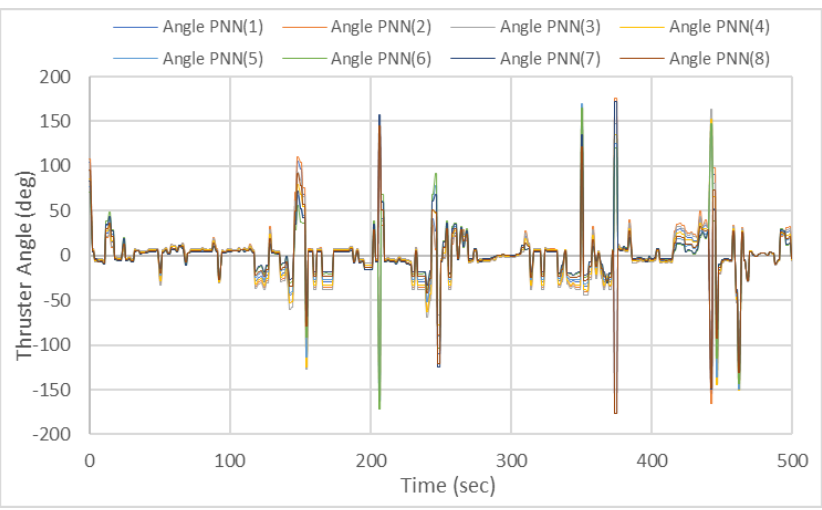

(d)

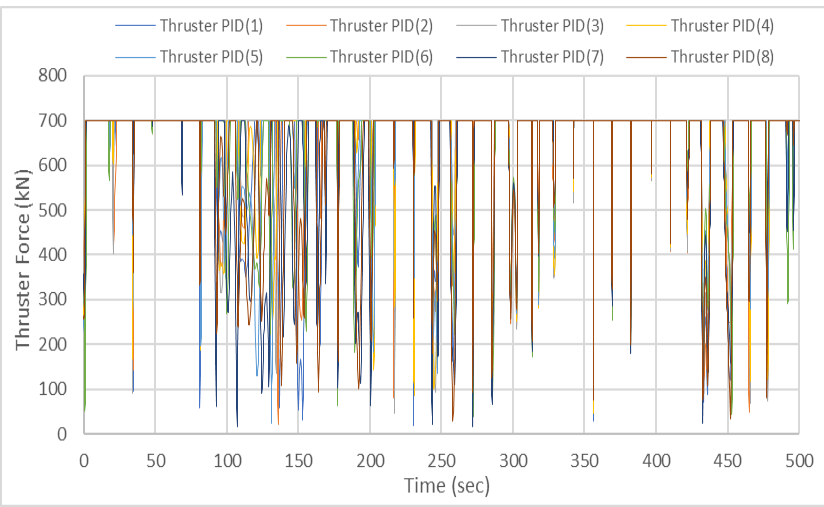

(e)

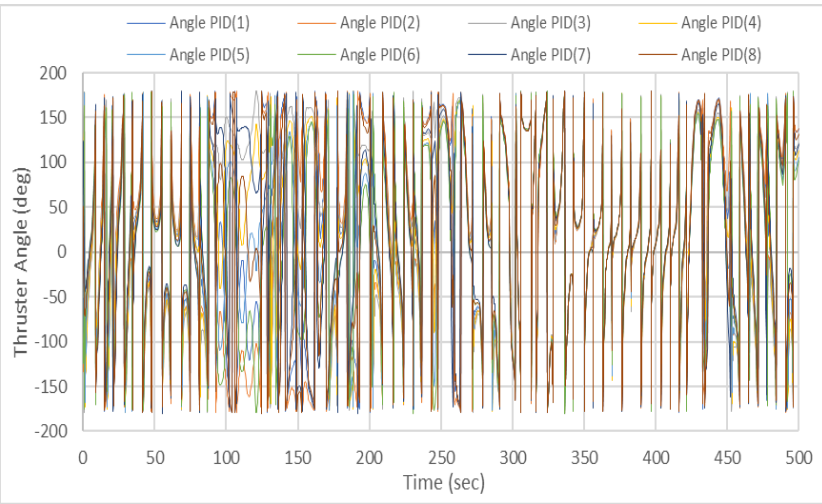

(f)

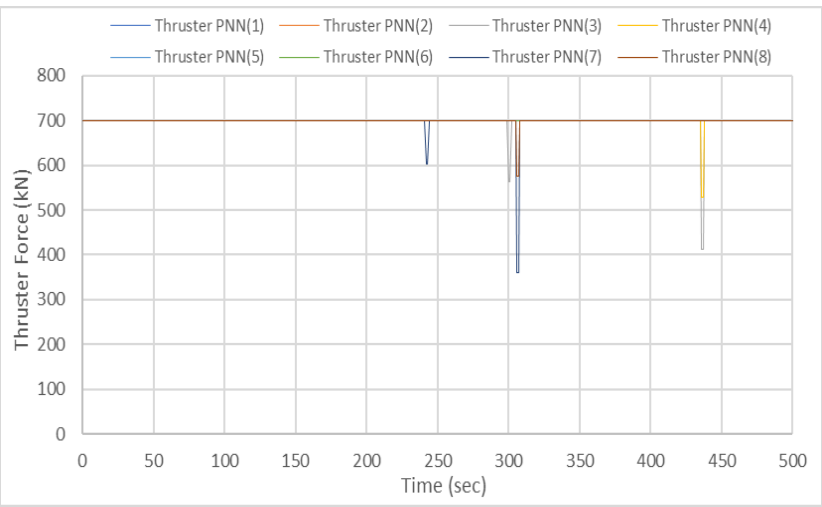

(g) 


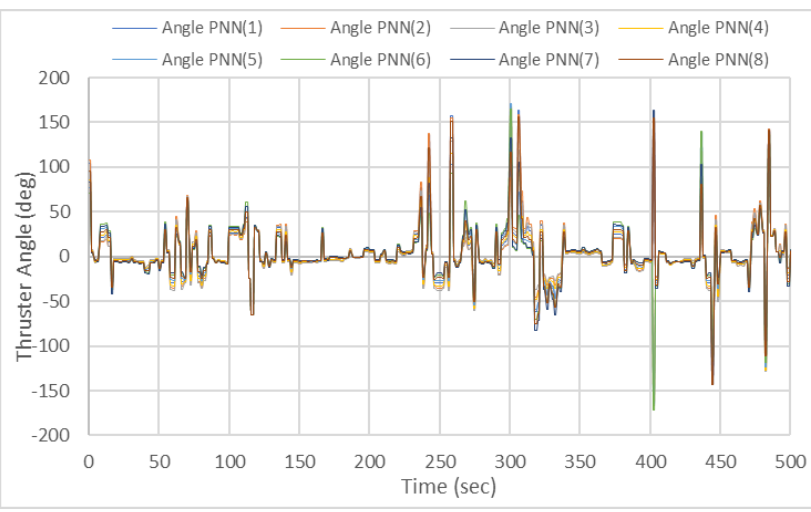

(k)

FIGURE 9: THRUSTERS FORCE AND ANGLE WITH PID AND PNN CONTROLS FOR HEAD SEA (A-D) AND BEAM SEA (E-K).

TABLE 7: STANDARD AVERAGE AND DEVIATION ANALYSIS OF THRUSTERS' FORCES AND ANGLE

\begin{tabular}{|c|c|c|c|c|}
\hline $\begin{array}{c}\text { Thruster } \\
\text { No. }\end{array}$ & $\begin{array}{c}\text { Average } \\
\text { Thrust (kN) }\end{array}$ & $\begin{array}{c}\text { SD Thrust } \\
(\mathrm{kN})\end{array}$ & $\begin{array}{c}\text { Average } \\
\text { Angle (deg) } \\
\end{array}$ & $\begin{array}{l}\text { SD Angle } \\
\text { (deg) }\end{array}$ \\
\hline \multicolumn{5}{|c|}{ PID (head sea) } \\
\hline 1 & 619.46 & 149.17 & 48.99 & 66.30 \\
\hline 2 & 619.08 & 149.67 & 48.07 & 65.88 \\
\hline 3 & 617.33 & 146.51 & 49.69 & 67.89 \\
\hline 4 & 611.51 & 150.59 & 50.12 & 67.70 \\
\hline 5 & 618.77 & 143.64 & 53.97 & 71.05 \\
\hline 6 & 630.21 & 136.60 & 57.75 & 74.57 \\
\hline 7 & 639.02 & 129.98 & 56.78 & 73.82 \\
\hline 8 & 629.10 & 136.83 & 52.85 & 70.07 \\
\hline \multicolumn{5}{|c|}{ PID and PNN (head sea) } \\
\hline 1 & 700.00 & 0.00 & 18.51 & 31.86 \\
\hline 2 & 700.00 & 0.00 & 19.99 & 33.71 \\
\hline 3 & 700.00 & 0.00 & 20.39 & 34.55 \\
\hline 4 & 700.00 & 0.00 & 18.96 & 32.85 \\
\hline 5 & 700.00 & 0.00 & 17.17 & 31.14 \\
\hline 6 & 700.00 & 0.00 & 15.29 & 29.65 \\
\hline 7 & 700.00 & 0.00 & 14.95 & 28.99 \\
\hline 8 & 700.00 & 0.00 & 16.74 & 30.12 \\
\hline $\begin{array}{c}\text { Thruster } \\
\text { No. }\end{array}$ & $\begin{array}{c}\text { Average } \\
\text { Thrust (kN) }\end{array}$ & $\begin{array}{c}\text { SD Thrust } \\
(\mathrm{kN})\end{array}$ & $\begin{array}{c}\text { Average } \\
\text { Angle (deg) }\end{array}$ & $\begin{array}{c}\text { SD Angle } \\
\text { (deg) }\end{array}$ \\
\hline \multicolumn{5}{|c|}{ PID (quarter sea) } \\
\hline 1 & 606.51 & 157.52 & 52.81 & 70.73 \\
\hline 2 & 628.36 & 130.75 & 60.89 & 77.20 \\
\hline 3 & 628.03 & 136.77 & 59.18 & 75.16 \\
\hline 4 & 608.34 & 159.57 & 50.66 & 68.23 \\
\hline 5 & 613.79 & 154.33 & 50.45 & 69.04 \\
\hline 6 & 637.44 & 122.48 & 59.99 & 77.06 \\
\hline 7 & 626.70 & 132.41 & 60.12 & 77.20 \\
\hline 8 & 603.56 & 162.54 & 51.51 & 69.93 \\
\hline \multicolumn{5}{|c|}{ PID and PNN (quarter sea) } \\
\hline 1 & 700.00 & 0.00 & 19.85 & 32.70 \\
\hline 2 & 699.60 & 6.31 & 19.35 & 31.46 \\
\hline 3 & 699.60 & 6.31 & 19.50 & 31.26 \\
\hline 4 & 700.00 & 0.00 & 19.99 & 32.45 \\
\hline 5 & 700.00 & 0.00 & 20.60 & 33.75 \\
\hline 6 & 700.00 & 0.00 & 21.15 & 35.12 \\
\hline 7 & 700.00 & 0.00 & 21.25 & 35.54 \\
\hline 8 & 700.00 & 0.00 & 20.58 & 34.18 \\
\hline
\end{tabular}

\begin{tabular}{ccccc}
\hline $\begin{array}{c}\text { Thruster } \\
\text { No. }\end{array}$ & $\begin{array}{c}\text { Average } \\
\text { Thrust (kN) }\end{array}$ & $\begin{array}{c}\text { SD Thrust } \\
(\mathbf{k N})\end{array}$ & $\begin{array}{c}\text { Average } \\
\text { Angle (deg) }\end{array}$ & $\begin{array}{c}\text { SD Angle } \\
\text { (deg) }\end{array}$ \\
\hline \multicolumn{5}{c}{ PID (beam sea) } \\
\hline $\mathbf{1}$ & 628.44 & 140.71 & 83.90 & 100.13 \\
\hline $\mathbf{2}$ & 649.39 & 114.74 & 93.18 & 107.89 \\
\hline $\mathbf{3}$ & 652.29 & 113.06 & 82.57 & 97.59 \\
\hline $\mathbf{4}$ & 637.14 & 128.83 & 73.09 & 89.28 \\
\hline $\mathbf{5}$ & 629.74 & 129.01 & 72.04 & 86.93 \\
\hline $\mathbf{6}$ & 629.28 & 127.94 & 76.22 & 90.49 \\
\hline $\mathbf{7}$ & 606.44 & 161.16 & 80.28 & 95.20 \\
\hline $\mathbf{8}$ & 611.52 & 150.12 & 80.02 & 96.24 \\
\hline \multicolumn{5}{r}{ PID and PNN (beam sea) } \\
\hline $\mathbf{1}$ & 700.00 & 0.00 & 19.94 & 32.65 \\
\hline $\mathbf{2}$ & 700.00 & 0.00 & 19.66 & 32.26 \\
\hline $\mathbf{3}$ & 698.31 & 20.06 & 19.07 & 31.27 \\
\hline $\mathbf{4}$ & 699.32 & 10.80 & 19.27 & 31.18 \\
\hline $\mathbf{5}$ & 700.00 & 0.00 & 19.23 & 31.14 \\
\hline $\mathbf{6}$ & 700.00 & 0.00 & 19.03 & 31.25 \\
\hline $\mathbf{7}$ & 698.25 & 22.35 & 19.61 & 31.81 \\
\hline $\mathbf{8}$ & 699.50 & 7.83 & 19.97 & 32.65 \\
\hline
\end{tabular}

The DP simulation using PID control only with head, quarter and beam sea angles of attack is shown in Figures 6-8(a) in which it is clear that the control has difficulty maintaining the platform within the limit of the drilling zone in this harsh weather, and that it lost the ability to maintain the position and heading under the head and beam sea conditions. Also, the thruster force and angle efficiency show potential high wear and tear with maximum forces and large thrust directions, as shown in Figures 9(a-b, e-f). Meanwhile, the DP system using PID and PNN controls is able to maintain the position and heading in this extreme sea condition, but not in the safe green zone to continue the drilling operations, as shown in Figures 6-8(b). Besides, the thruster forces and angle efficiency illustrate that the forces and directions are more stable and with small angle deviation, and therefore less wear and tear of the thrusters is likely with the forces applied with directions \pm 40 degree which minimizes the thrust loss effect as shown in Figures 9(c-d, g-k).

The DP simulation results show a faster response, shorter peak and settling time and less overshoot of the set-position and heading using PNN control, with head, quarter and beam sea angles of attack are shown in Tables 4-6. In addition, the average and standard deviation results from statistical analysis of the thruster force and angle clearly show that the PID plus PNN has a higher force average and lower angle average, and lower standard deviation for both force and angle as shown in Table 7.

In the simulation, the PID motion control was not able to respond fast enough to maintain the position and heading of the platform. The use of the computationally-efficient PNN control to minimize the cost function can be observed. The PNN allocation control improved the position of the platform and heading performance by sufficiently distributing the thruster forces at smaller angles than in the PID motion control case. The results showed that the PNN algorithm can efficiently stabilize the force and direction of the thrusters. The position and heading accuracy improvement percentage using PNN allocation control as compared to using PID alone is shown in Table 8. 
TABLE 8: POSITION AND HEADING ACCURACY IMPROVEMENTS PERCENTAGE USING PNN CONTROL.

\begin{tabular}{cccc}
\hline $\begin{array}{c}\text { Environmental } \\
\text { Attack Angle }\end{array}$ & $\begin{array}{c}\mathbf{X} \text { position } \\
(\boldsymbol{\%})\end{array}$ & $\begin{array}{c}\text { Y position } \\
(\boldsymbol{\%})\end{array}$ & $\begin{array}{c}\text { Heading } \\
(\boldsymbol{\%})\end{array}$ \\
\hline Head sea & 19.35 & 53.69 & 1 \\
\hline Quarter sea & 25.69 & -80.97 & 5.68 \\
\hline Beam sea & -85.37 & 27.62 & 8.54 \\
\hline
\end{tabular}

The results show that the semi-submersible platform could maintain the position and heading in the safe drilling zone under different environmental angles of attack by using the combination of PID control for motion and PNN control to organize the thruster forces. The simulation found clear evidence that PID control alone could not handle this high sea condition of sea state seven or more and needed the PNN control to increase the accuracy to maintain safe operations. Additionally, the PID control would potentially cause significant wear and tear in terms of the thruster forces and directions. In contrast, the PNN control can minimize this through appropriate thruster allocation. Although drilling would not be expected to continue in such extreme conditions, one of the targets of the future work is to extend the weather window in which drilling can take place. Control technology is progressing with the trend towards intelligent control and increased reliability.

\section{CONCLUSION}

The hull of an existing semi-submersible drilling platform was modelled according to DNV standards using DNV Sesam (GeniE and HydroD), SIMO and WAMIT tools. This paper improved and developed the accuracy of the DP thruster allocation control by adding a PNN algorithm to the existing basic DP system, enhancing the safety and zone keeping of DP operations in harsh weather condition. The PNN can keep position under 50 years probability harsh weather whereas PID cannot maintain the platform in the drilling safe zone. It shows that the PNN control, implementing artificial intelligence into the thruster allocation, is more reliable than requiring the DP operator to manage all of the drilling operations independently. In addition, the PID control strategy implemented alone has a higher failure rate due to the thruster performance requirement. Further studies are required to develop the PNN thruster allocation control performance using advanced motion control such as sliding mode and back-stepping controls, taking into account the effect of the semi-submersible environmental loads and the drilling safety zone tightness regarding the water depth. New prediction approaches such as nonlinear automatic regression with recessive exogenous (NARX) will be investigated in the thruster allocation control.

\section{ACKNOWLEDGEMENTS}

The first author would like to express his deep gratitude to his research supervisors for their patient guidance and support of this research work. Special thanks are also extended to the Faculty of Maritime Studies, King Abdul-Aziz University, for sponsoring the research.

\section{REFERENCES}

[1]. Fay, H. Dynamic Positioning System Principles, Design and Applications, Paris: Technip, 1990.

[2]. Sørensen, A.J. A survey of dynamic positioning control systems, Annu. Rev. Control, 35, 123-136, 2011.

[3]. Mehrzadi, M. Terriche, Y. Su, C.L. Othman, M.B. Vasquez, J.C. Guerrero, J.M. Review of Dynamic Positioning Control in Maritime Microgrid Systems, Energies, 13, 3188, 2020.

[4]. Johansen, T.A. Fossen, T.I. Control allocation-a survey, Automatica, 49(5):1087-1103, 2013.

[5]. Hauff, K. Analysis of Loss of Position Incidents for Dynamically Operated Vessels, Department of Marine Technology, Norwegian University of Science and Technology (NTNU), 2014.

[6]. Fossen, T.I. Guidance and Control of Ocean Vehicles, Wiley: Hoboken, NJ, USA, 1994.

[7]. Fossen, T.I. Handbook of Marine Craft Hydrodynamics and Motion Control, IEEE Control Syst. 2011.

[8]. Det Norske Veritas (DNV). Sesam User Manual GeniE and HydroD, Det Norske Veritas (DNV) Software, 2014.

[9]. WAMIT. WAMIT User Manual Version 7.2, WAMIT software, Massachusetts Institute of Technology, WAMIT, Inc 2016.

[10]. Sørensen, A.J. Marine Control Systems: Propulsion and Motion Control of Ships and Ocean Structures, Department of Marine Technology, Norwegian University of Science and Technology (NTNU), 2013.

[11]. Faltinsen, O, M. Sea Loads on Ships and Offshore Structures, Cambridge University Press, 1990.

[12]. Chin, C.S. Dynamic positioning simulation, thrust optimization design and control of a drill ship under disturbances and faulty thruster, The society for modelling and simulation international, pp. 1338-1349, doi: 10.1177/0037549712450345, 2012.

[13]. Fossen, T.I. Perez, T. Marine Systems Simulator (MSS), URL: https://github.com/cybergalactic/MSS, 2004.

[14]. Soloway, D. Haley, P.J. Neural generalized predictive control, Proceedings of the 1996 IEEE International Symposium on Intelligent Control, Dearborn, MI, USA, pp. 277-282, doi: 10.1109/ISIC.1996.556214, 1996.

[15]. HSE. Environmental considerations, Health \& Safety Executive, ISBN 071762379 3, 2002.

[16]. Chen, H. Moan, T. Verhoeven, H. Safety of Dynamic Positioning Operation on Mobile Offshore Drilling Units, Reliability Engineering and System Safety Journal, 2008. 